\title{
A Design Methodology for Energy-Aware Processing in Unmanned Aerial Vehicles
}

\author{
JINGYU HE and YAO XIAO, University of Southern California \\ CORINA BOGDAN, Northeastern University \\ SHAHIN NAZARIAN and PAUL BOGDAN, University of Southern California
}

\begin{abstract}
Unmanned Aerial Vehicles (UAVs) have rapidly become popular for monitoring, delivery, and actuation in many application domains such as environmental management, disaster mitigation, homeland security, energy, transportation, and manufacturing. However, the UAV perception and navigation intelligence (PNI) designs are still in their infancy and demand fundamental performance and energy optimizations to be eligible for mass adoption. In this article, we present a generalizable three-stage optimization framework for PNI systems that (i) abstracts the high-level programs representing the perception, mining, processing, and decision making of UAVs into complex weighted networks tracking the interdependencies between universal low-level intermediate representations; (ii) exploits a differential geometry approach to schedule and map the discovered PNI tasks onto an underlying manycore architecture. To mine the complexity of optimal parallelization of perception and decision modules in UAVs, this proposed design methodology relies on an Ollivier-Ricci curvature-based load-balancing strategy that detects the parallel communities of the PNI applications for maximum parallel execution, while minimizing the inter-core communication; and (iii) relies on an energyaware mapping scheme to minimize the energy dissipation when assigning the communities onto tile-based networks-on-chip. We validate this approach based on various drone PNI designs including flight controller path planning, and visual navigation. The experimental results confirm that the proposed framework achieves $23 \%$ flight time reduction and up to $34 \%$ energy savings for the flight controller application. In addition, the optimization on a 16-core platform improves the on-time visit rate of the path planning algorithm by $14 \%$ while reducing $81 \%$ of run time for ConvNet visual navigation.
\end{abstract}

CCS Concepts: • Networks $\rightarrow$ Network on chip; • Theory of computation $\rightarrow$ Graph algorithms analysis; • Computer systems organization $\rightarrow$ Embedded hardware; Multicore architectures;

Additional Key Words and Phrases: Unmanned aerial vehicles, complex networks, community detection, ollivier-ricci curvature, load balancing, pipeline parallelism, manycore systems

The authors gratefully acknowledge the support by the National Science Foundation under the Career Award CPS/CNS1453860, the NSF award under Grant Numbers CCF-1837131, MCB-1936775, CNS-1932620, and CMMI 1936624, the DARPA Young Faculty Award and DARPA Director's Fellowship Award, under Grant Number N66001-17-1-4044, and a Northrop Grumman grant. The views, opinions, and/or findings contained in this article are those of the authors and should not be interpreted as representing the official views, or policies, either expressed, or implied by the Defense Advanced Research Projects Agency, the Department of Defense, or the National Science Foundation.

Authors' addresses: J. He, Y. Xiao, S. Nazarian, and P. Bogdan, University of Southern California, 3740 McClintock Ave, Los Angeles, CA 90007; emails: \{jingyuhe, xiaoyao, shahin.nazarian, pbogdan\}@usc.edu; C. Bogdan, Northeastern University, 360 Huntington Ave, Boston, MA 02115; email: i.bogdan@northeastern.edu.

Permission to make digital or hard copies of all or part of this work for personal or classroom use is granted without fee provided that copies are not made or distributed for profit or commercial advantage and that copies bear this notice and the full citation on the first page. Copyrights for components of this work owned by others than ACM must be honored. Abstracting with credit is permitted. To copy otherwise, or republish, to post on servers or to redistribute to lists, requires prior specific permission and/or a fee. Request permissions from permissions@acm.org.

(C) 2021 Association for Computing Machinery.

1084-4309/2021/09-ART4 \$15.00

https://doi.org/10.1145/3470451

ACM Transactions on Design Automation of Electronic Systems, Vol. 27, No. 1, Article 4. Pub. date: September 2021. 
ACM Reference format:

Jingyu He, Yao Xiao, Corina Bogdan, Shahin Nazarian, and Paul Bogdan. 2021. A Design Methodology for Energy-Aware Processing in Unmanned Aerial Vehicles. ACM Trans. Des. Autom. Electron. Syst. 27, 1, Article 4 (September 2021), 20 pages.

https://doi.org/10.1145/3470451

\section{INTRODUCTION}

Unmanned aerial vehicles (UAVs) are emerging as critical tools for numerous applications that involve executing dangerous (to humans) tasks in evolving, uncertain and extreme conditions. UAVs may also have the potential to significantly reduce the time and resources needed for certain applications and even save human lives. For example, drones have been used for aerial monitoring, evaluation, and delivery missions in the COVID-19-affected areas [32]. From the traditional quadrotors [18] to a fully actuated hexarotors [30], and to the futuristic volocopoters [37], many researchers have presented various designs of the UAV architectures for different application scenarios (see Figure 1). The computational requirements increase quadratically as the number of propellers rises. As later illustrated in Equation (1), an addition of a pair of propellers brings an extra dimension to the thrust vector and torque vector of the dynamic of the UAV, making the matrix multiplication of the responsive flight controller more demanding from a computational perspective. The increasingly complicated self-navigation and collision-avoidance applications of the evolving UAVs call for a high-performance and low-power flight controller.

The embedded systems on UAVs are responsible for both analyzing the visual information detected via different sensors and calculating the flight dynamics under tight budgets of energy and computing power. The UAV PNI applications are highly demanding in terms of energy efficiency and computing power. To address these issues, tile-based network-on-chip (NoC) [1, 10, 25] is the ideal distributed computing architecture for scalable and low-power on-chip communication. As shown in a report published by the International Technology Roadmap for Semiconductors (ITRS), the multi-processor systems-on-chips (MPSoCs) will integrate thousands of processors [15] by 2025, making the conventional time-division multiplexing bus-based MPSoCs no longer feasible due to its poor scalability. Although integrated Central Processing Unit (CPU) and Graphics Processing Unit (GPU) platforms have been widely used in UAVs, there is still a need for integrating the video encoder, modem, flight controller IP, and other critical components (e.g., UAV-to-UAV communication, UAV-to-infrastructure, UAV-to-network, and UAVto-cloud), which are mostly separate chips right now, into a single-chip solution for the next generation drones. The $\mathrm{NoC}$ is the ideal communication solution that is able to combine several drone functions and addresses important drone design issues such as power consumption and complexity at the same time. The tile-based NoC's packet routing mechanism makes it highly scalable for integrating large number of heterogeneous cores. As shown in Figure 2, usually, the NoC architectures consist of a grid of tiles which could encapsulate one or more CPUs, GPUs, Application-Specific Integrated Circuits (ASICs), and so on. Applications are scheduled and mapped onto each tile based on their computational requirements and timing characteristics. Cores in various tiles communicate via designated routers and following specific communication protocols $[1,24]$. There have been many previous studies on energy-aware NoC designs. In contrast to the prior NoC work, the goal of this article is to investigate the parallelization of the UAV perception and navigation intelligence (PNI) while considering the computation and communication power consumption. We have therefore analyzed the characteristics of UAV PNI applications and designed a framework that realizes those characteristics. As shown in Figure 3, we first compile the PNI programs into Low-Level Virtual Machine (LLVM) Intermediate Representation (IR) and construct the data dependency graph (DDG), where each node denotes only a useful low-level 


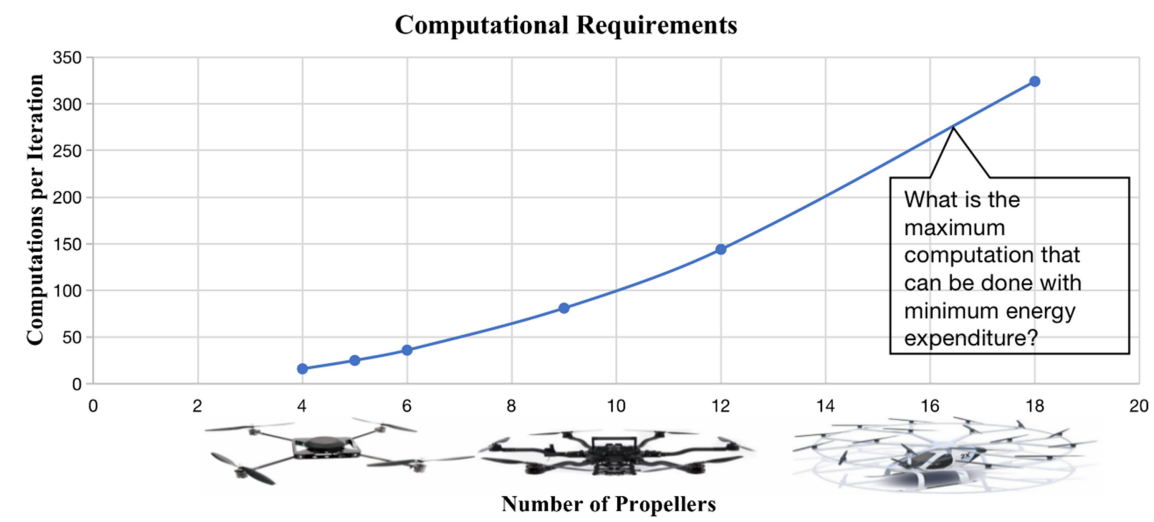

Fig. 1. Various multirotos and their computational requirements: quadrotor (left), hexarotor (middle), and volocoptor (right). Increasing the number of rotors can provide not only higher elevation power and trust, contributing to higher safety, stability, robustness, and resilience to turbulent flows or other perturbations, but also requires significant computational (processing) capabilities for precise control and maneuvering or coordination across the UAV components. These computational requirements can only be exacerbated when considering the need for accounting for UAV-to-UAV communication, trustworthiness evaluation, and other decision making in crowded environments.
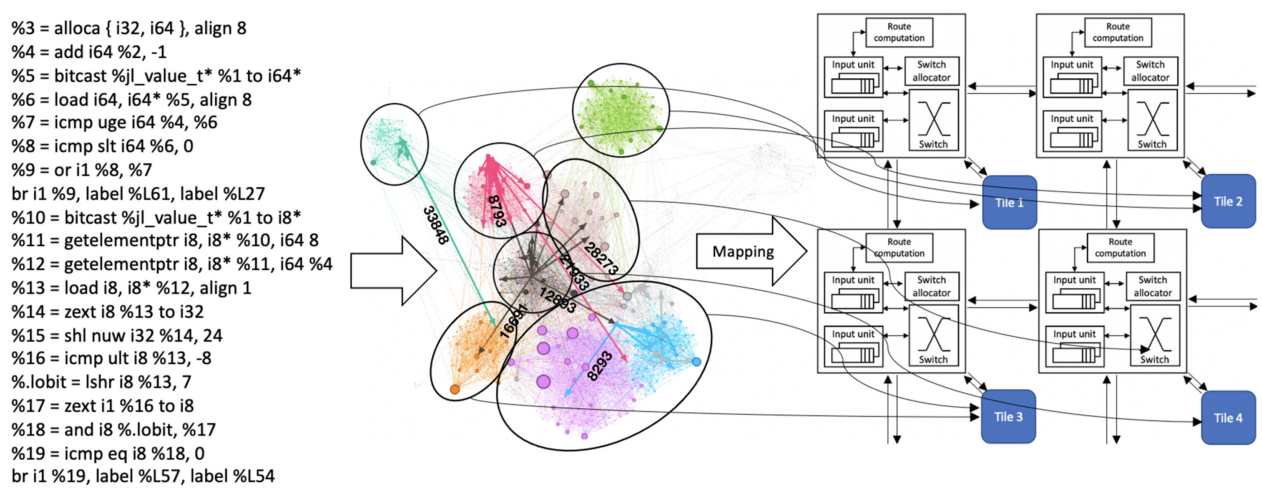

Fig. 2. Tile-based NoC architecture. A drone high-level code application is profiled and transformed into a weighted graph, where each node represents a universal low-level instruction and each edge represents a dependency between instructions. The thickness of the edge is proportional to the data size and the size of the node is related to the degree of the node. Selected major dependencies are marked with their data size. Finally, a closely related group of instructions is mapped onto the tiles of the NoC architecture.

instruction (assumed to run an universal machine and avoiding specific instruction set architectures) and each edge represents the data dependency whose weight denotes the data size times latency. Second, based on the DDG, we propose a community detection and an Ollivier-Ricci curvature (ORC)-based scheduling algorithm to partition the PNI applications into clusters and map them onto a manycore architecture such that (1) the inter-cluster communication is minimized; (2) the NoC energy is reduced; and (3) the workloads of different cores are balanced for maximum parallel execution. Finally, we incorporate a topological sort into our energy-aware mapping scheme to further reduce the static power consumption resulted from congestion.

Toward this end, the main contributions of this article are as follows: 

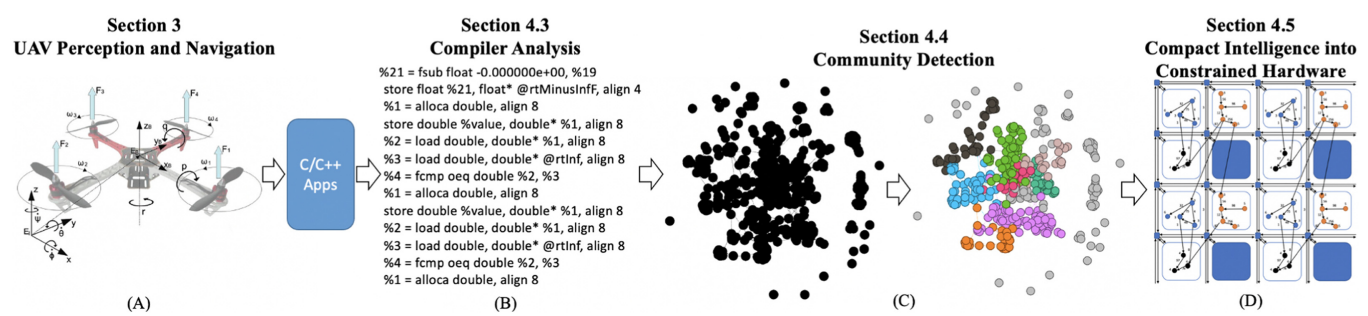

Fig. 3. Overview of the UAV intelligent processing architecture design methodology. (A) An UAV and its control basics. (B) The PNI application (as a high-level program) is compiled into LLVM IR trace through compiler analysis. This allows us to remove the unnecessary computation and communication overhead of high-level programs. (C) We transform the trace into the DDG by extracting the computation model and identify the communities (different communities are coded with different colors) through a differential geometry approach that relies on the ORC concept. (D) Each processing community is mapped onto a processing element of the underlying manycore system such that its communication energy is minimized and congestion is reduced. The unused cores are clock-gated to save energy, indicated by the blue tiles.

-We develop a novel design and optimization framework that (i) utilizes a graph modeling approach to capture the task dependencies of PNI applications; (ii) partitions the PNI tasks based on community detection ideas and maps them onto an underlying NoC- based manycore systems based on a differential geometry algorithmic strategy, to minimize intercluster communication and energy, and maximize parallelism considering the characteristics of ultra-low-power embedded UAV applications.

-We put our framework to test using several UAV perception and navigation applications and report that it achieves significant performance speedup and energy savings against the state-of-the-art flight controller.

The rest of this article is organized as follows: Section 2 presents the related work. Section 3 introduces the basics of UAV control, parallelism, and community detection. Section 4 illustrates the energy model for our manycore systems, the ORC-based load-balancing, energy-aware community detection, and low-power mapping algorithms. Section 5 validates the framework and shows experimental results compared to the baseline model.

\section{RELATED WORK}

As UAV's core component, the avionic is a system that integrates hardware and software to make drones fully capable of flying without human beings' guidance. The complex avionic applications have fixed deadlines and can benefit from running their codes on multicore systems. In this section, we offer a brief survey of avionics design on both bus- and NoC-based MPSoCs.

\subsection{Bus-Based MPSoCs}

Spatial and temporal partitioning are two types of partitioning for bus-based MPSoCs. Spatial partitioning refers to the separation of resources among different applications. The hybrid architecture proposed by Avramenko et al. [4] uses a hypervisor to avoid low-criticality applications from corrupting the high-criticality applications. Esposito et al. [12] designed a system that allows the consolidation of different applications with different safety and performance requirements on the same chip. On the other hand, temporal partitioning allows different applications to share core resources in a time-multiplexing way. The interference-sensitive worst-case execution time (WCET) analysis is applied in $[27,28]$ to consider the number of cores that are accessing a shared resource while evaluating the access latency. 


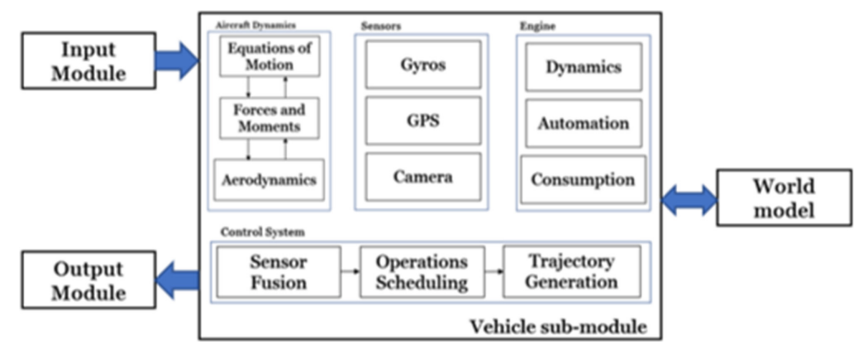

Fig. 4. The block diagram of a typical UAV control system. We have chosen three applications from the aerial vehicle sub-module to apply our design methodology. The flight controller is a part of the drone dynamics, while the path planning algorithm of the control system generates the trajectory. Lastly, the visual navigation application analyses the data captured by the camera sensor for obstacle avoidance.

\subsection{NoC-Based MPSoCs}

To address the increasing computational and energy demand of UAV's avionics, the focus has recently moved from bus-based multi-core systems to NoC-based MPSoCs. An important concept for NoC-based MPSoCs is the partitioning and scheduling. To overcome the limitation of the workload models in [6, 7] and capture the intricate computational interdependencies, Xiao et al. [39] described a complex network inspired modeling of applications and partitioning scheme for multicore design. Along the same lines, the work in [8] proposed a task assignment algorithm to allocate a valid processor and start time for each task and get an upper bound on the number of processors required by the tasks. Similarly, the work in [14] presented a task clustering algorithm which hierarchically merges the Hungarian scheduling. Tariq et al. proposed an Integer Linear Programming (ILP)-based scheduling and mapping algorithm in [36] for NoC-based MPSoCs. Tan et al. developed a low-power customizable manycore architecture using a lightweight message-passing scheme [35].

In contrast to prior work, we present an energy-aware load-balancing community detection algorithm together with a mapping strategy and test it using several UAV self-navigation applications. This proposed methodology is general and can be extended to future swarms of UAVs that will exhibit significant computational requirements for perception, interaction/communication with other UAVs and infrastructure, planning, and decision making activities.

\section{PRELIMINARIES}

In this section, we first provide a background description of the three UAV PNI benchmark applications. Figure 4 shows the block diagram of a typical UAV control system. We have chosen three applications from the aerial vehicle sub-module to apply our design methodology, including the UAV flight controller, the path planning algorithm, and the visual navigation computational modules. Next, we introduce the preliminaries of our optimization framework, such as the pipeline parallelism and the community detection in weighted graphs.

\subsection{Quadrotor Flight Controller}

Figure 3(A) shows a UAV with six degrees of freedom. Three degrees of freedom describe the translational motions $(x, y, z)$ and the other three are the rotational motions $(r, p, q)$. Each of the four propellers is equipped with a rotor providing the angular velocity. These four angular velocities correspond to the inputs of the quadrotor, $\omega_{i}=\left[\omega_{1}, \omega_{2}, \omega_{3}, \omega_{4}\right]$. A total of 12 outputs are generated from the quadrotor, $X=[x, y, z, r, p, q, \dot{x}, \dot{y}, \dot{z}, \dot{r}, \dot{p}, \dot{q}]$, corresponding to the translational and rotational positions, and their corresponding velocities [9]. 
For real-time applications, the error between the actual UAV position, estimated by a navigation system, and the desired position is fed into a PD-controller to determine the required control inputs. The required rotor speeds are then calculated from the respective torques using the following equation:

$$
\left(\begin{array}{l}
T \\
\Gamma
\end{array}\right)=\left(\begin{array}{cccc}
-b & -b & -b & -b \\
0 & -d b & 0 & d b \\
-d b & 0 & d b & 0 \\
k & -k & k & -k
\end{array}\right)\left(\begin{array}{c}
\omega_{1}^{2} \\
\omega_{2}^{2} \\
\omega_{3}^{2} \\
\omega_{4}^{2}
\end{array}\right),
$$

where $T$ is the thrust vector for each propeller, $\Gamma$ is the torque vector applied to the airframe, $b$ represents the lift constant, $d$ is the distance from the rotor to the center of the mass, and $k$ is secondary lift constant. The control structure employed to fly the quadrotor can be found in $[3,9]$, and is based on Proportional Derivative action to get the quadrotor's attitude (roll, pitch, yaw) and altitude.

\subsection{UAV Path Planning}

The path planning [29] problem seeks to optimize the route of a UAV that starts from an initial position, visits several sites of interest for accomplishing certain tasks, avoids unsafe areas, and goes to an end position. The path needs to satisfy certain physical constraints, e.g., maximum and minimum speed, maximum acceleration, maximum and minimum altitude, and minimum turning radius. The objectives of the optimization are two-fold: to minimize the energy consumption of the UAV and to maximize the reward for finishing tasks under timing constraints. For instance, in a natural disaster situation, UAVs could be used as an ideal tool to deliver delay-sensitive information between a rescue command center and thousands of civilians scattered over large remote areas. The path planning problem has been broadly studied under the name of Traveling Salesman Problem (TSP) [17, 31].

\subsection{UAV Visual Navigation}

The key driver for the visual navigation benchmark is DroNet [23]: an algorithm proposed by Loquercio et al. based on a convolutional neural network (CNN) whose topology is inspired on ResNet [16]. DroNet is trained to convert an unprocessed input image from a camera into two pieces of information: (i) an estimation of the probability of collision with an obstacle, which, in turn, can be used to determine the forward target velocity of the UAV; and (ii) the desired steering direction, following visual cues from the camera such as the presence of obstacles, white lines on the floor or on the street. Figure 5 shows the topology of DroNet, which is shared by the probability of collision and the steering angle. Udacity ${ }^{1}$ and Zurich Bicycle ${ }^{2}$ datasets are used to train steering and collision, respectively.

\subsection{Community Detection Analysis through Differential Geometry Concepts}

The community structures [26] refer to the grouping of nodes with a high density of edges within each community and sparse connections among clusters. It is of great significance to detect these community structures for which scientists can find self-similarity of groups of nodes, for example, in social networks. In many real-world settings, we lack knowledge about the number, size, and topological characteristics of the community structure of a given graph. To overcome these challenges, the ORC-based community detection [34] has been proposed and proven to be more

\footnotetext{
${ }^{1}$ https://www.udacity.com/self-driving-car.

${ }^{2}$ http://rpg.ifi.uzh.ch/dronet.html.
} 


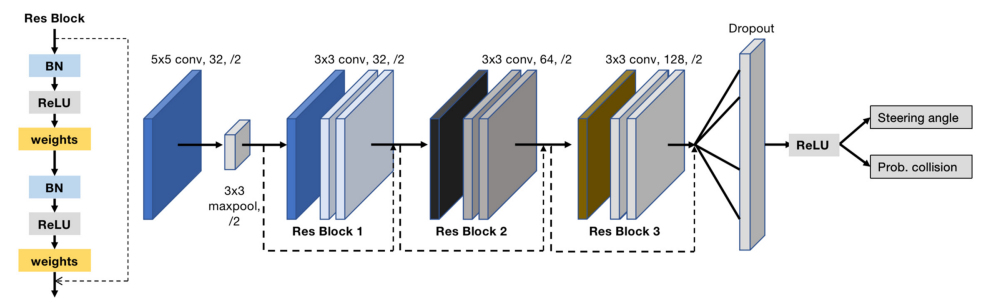

Fig. 5. DroNet architecture used for UAV visual navigation applications.

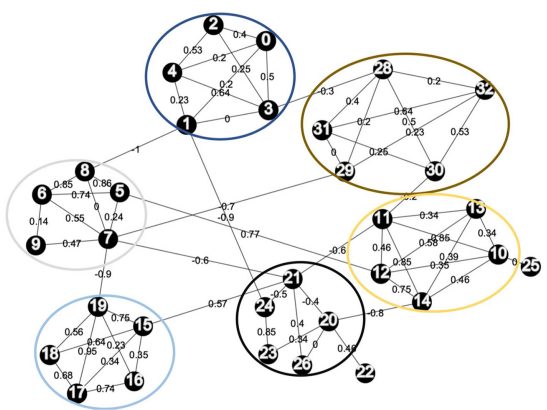

Fig. 6. ORC-based community detection. An artificial complex network is generated by the stochastic block model with the following parameters: size per community $k=5$, number of community $l=6$, and with two leaf nodes $(22,25)$.

efficient than modularity-based techniques [13] even when the communities are not densely connected and even for small community sizes. The notion of curvature, as defined in Riemannian geometry, quantifies how geodesic paths converge $(\mathrm{ORC}>0)$ or diverge $(\mathrm{ORC}<0)$. As an example, in Figure 6, each node represents an instruction and each edge corresponds to a dependency. The instructions in the same code block across iterations are grouped into communities. The values attached to the edges represent the ORC values. The exact computation of the ORC can be computed by exploiting optimal transport theory concepts and estimating the Wasserstein distance through a linear programming approach [38] or its parallel computation strategy [22]. The ORC captures the network flows of shortest paths via the Wasserstein's distance formulation where the positively curved edges are well connected and naturally form a community, while the negatively curved edges could be interpreted as "bridges" between communities and cutting them would isolate the network flow between communities.

Community detection strategies have not been exploited in parallel computing to discover the communities of intensive processing and inter-dependencies among instructions. This article proposes a new parallelization framework based on concepts from complex networks and differential geometry theories in order to obtain the optimal number of clusters with minimal inter-cluster edge weights based on the data-dependent structures of each UAV application.

\section{PARALLELIZATION DISCOVERY AND ENERGY OPTIMIZATION APPROACH}

\subsection{System Model}

In this article, we employ an NoC-based Chip Multiprocessor (CMP) architecture as shown in Figure 2. The architecture consists of multiple processor cores, and each core has its own data and program memory. If a task needs to read data that are not available in its local memory, inter- 
core communication happens. A router is embedded into each core for inter-core communication through NoC.

\subsection{Energy Model}

This work focuses on the energy consumption of the underlying manycore system. We present an energy model based on a cost function that captures the energy dissipation of both the IP cores and the on-chip interconnect. The cost function is used to evaluate our task mapping strategies. While most of the mapping algorithms based on the one in [19] only compute dynamic energy, our model considers both static and dynamic power dissipation.

Using the bit energy concept proposed by Ye et al. in [40], the total dynamic energy consumption can be computed by

$$
E_{D y N o C}=\sum_{i=1}^{a} \sum_{j=1}^{b} w_{i j}\left(\eta_{i j} E_{S_{b i t}}+\left(\eta_{i j}-1\right) \times E_{L_{b i t}}\right),
$$

where $E_{S_{b i t}}$ and $E_{L_{b i t}}$ represent the energy consumed by switch and link. Switch directs the packets and flits to their desired output ports and is the major consumer of the NoC's energy. A link connects a source router to a destination router and acts as a channel to transfer data between routers. $\eta_{i j}$ is the number of routers the packet from tile $\tau_{i}$ to tile $\tau_{j}$ passes through along the way; $w_{i j}$ is the size of the packet; and $a$ and $b$ denote the number of tiles on $x$ and $y$, respectively.

The static power is defined to characterize the energy consumed when packets are congested in the buffers. For simplicity, static power is defined as

$$
E_{S t N o C}=\sum_{i=1}^{n} P_{S t} \times w_{i} \times t_{i},
$$

where $n$ is the number of times that congestion occurs; $P_{S t}$ is the energy consumption of one bit of data stored in the buffer for one unit of time; $w_{i}$ is the data size of the $i$ th congestion; and $t_{i}$ is the time of the $i$ th congestion. Finally, Equation (4) gives the total energy consumption for the interconnect.

$$
E_{N o C}=E_{S t N o C}+E_{D y N o C} .
$$

\subsection{Compiler Analysis and Model of Computation Extraction}

To generate the DDG, we adopt the LLVM IR [20]. The rationale behind this is that LLVM is a language-independent system that exposes the commonly-used primitives to implement highlevel language features, which makes it very easy to generate back-end for any target platform.

With the help of Clang, $\mathrm{C} / \mathrm{C}++$ applications are compiled into a dynamic IR execution trace. We develop a parser to construct a DDG from the IR trace. The parser analyzes memory operations to obtain latency and data sizes. Because the execution times and energy vary on data sizes and where the data resides, taking those values into account could potentially reduce inter-core communications by grouping the source and destination instructions of a register into one cluster. Three hash tables are created and updated when parsing: the source table, the destination table, and the dependency table. The source/destination tables are used to keep track of source/destination registers with keys being source or destination registers and values being the corresponding line number. The dependency table is to store dependencies between nodes with keys being the line number for current instruction, and values being clock cycles, data sizes, and line numbers of previous instructions dependent on the same virtual register.

For example, in Figure 7, an LLVM IR snippet is extracted from an application compiled by Clang front-end and transformed into its corresponding DDG. As the parser reads the first line, a 
LLVM IR Trace
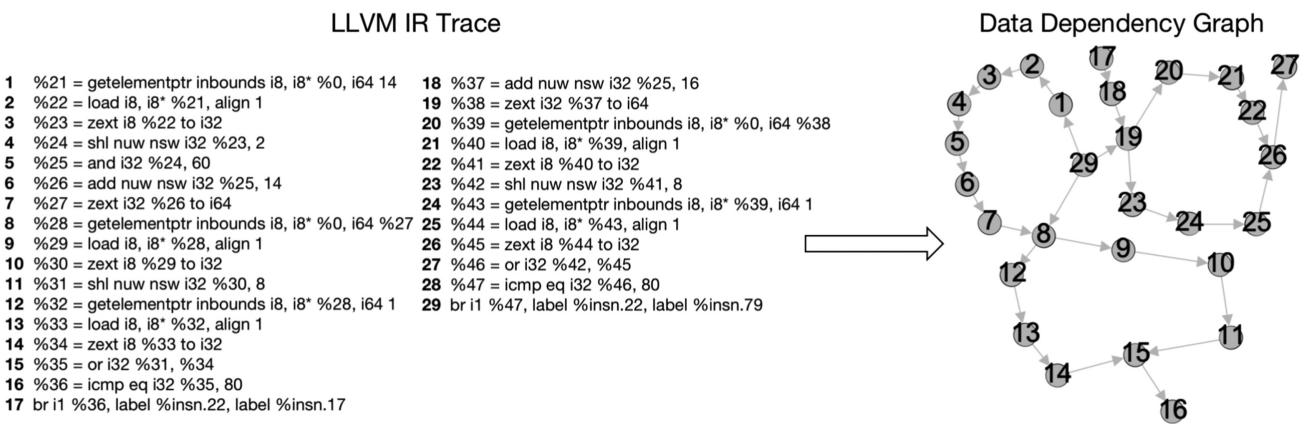

Fig. 7. An example of transformation from LLVM IR trace to DDG.

Table 1. The Source, Destination, and Weight Tables

\begin{tabular}{|c|c|c|c|c|c|}
\hline \multicolumn{2}{|c|}{ Src Table } & \multicolumn{2}{c|}{ Dest Table } & \multicolumn{2}{c|}{ Dependency Table } \\
\hline Key & Value & Key & Value & Key & Value \\
\hline$\% 0$ & 1 & $\% 21$ & 1 & 2 & $(1,8)$ \\
\hline$\% 21$ & 2 & $\% 22$ & 2 & 3 & $(2,8)$ \\
\hline$\% 22$ & 3 & $\% 23$ & 3 & 4 & $(3,32)$ \\
\hline$\% 23$ & 4 & $\% 24$ & 4 & 5 & $(4,32)$ \\
\hline$\% 24$ & 5 & $\% 25$ & 5 & 6 & $(5,32)$ \\
\hline$\% 25$ & 6 & $\% 26$ & 6 & 7 & $(6,32)$ \\
\hline$\% 26$ & 7 & $\% 27$ & 7 & 8 & $(7,64)$ \\
\hline$\% 27, \% 0$ & 8 & $\% 28$ & 8 & 9 & $(8,8)$ \\
\hline$\% 28$ & 9 & $\% 29$ & 9 & & \\
\hline
\end{tabular}

source table and a destination table are created in Table 1 . Please note that Table 1 only shows the dependency of the first nine lines to save space. The source table is updated with the key being $\% 0$ and the value being 1 and its destination register is hashed into the destination table with the key being $\% 21$ and the value being 1 . When parser reads line 2, the source register $\% 21$ happens to be the destination register in line 1. A dependency table is created and updated with the key being 2 (line number of current instruction) and value being $(1,8)$, a tuple where the first item is the line number of the dependent instruction and the second item is the data size. Following the same procedure, the three hash tables will look like what is shown in Table 1. And the corresponding DDG is generated in the right column in Figure 7. Please note that although the edge weight of the DDG is not shown in Figure 7, it can be calculated using the data size.

\subsection{Discovering the Processing Community Structure}

To formulate this problem, we introduce the following concepts:

Definition 4.1. A DDG is a weighted directed graph $G=G\left(a_{i}, b_{i j}, e_{i}, w_{i j}|i, j \in N|\right)$ where each vertex $a_{i}$ represents an LLVM IR instruction; each edge $b_{i j}$ with weights $w_{i j}$ characterizes either the dependency from $a_{i}$ to $a_{j}$ or the control flow such as jumps or branches from one block to another; and $e_{i}$ stands for the estimated energy of the vertex given in Section 4.1.

Definition 4.2. A weight $w_{i j}$ between $a_{i}$ and $a_{j}$ is calculated by latency times data size. Latency characterizes the delay from $a_{i}$ to $a_{j}$ based on the timing information of the memory operation provided by the compiler. Data size represents the number of bytes transferred. 


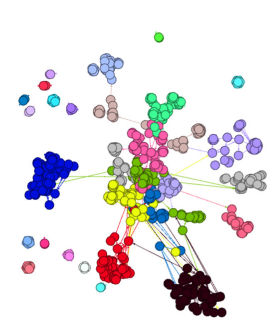

(a)

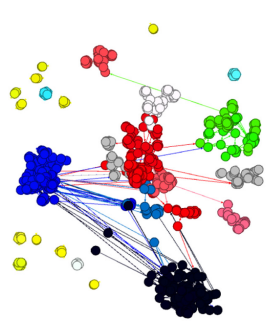

(b)

Fig. 8. The DDG is obtained from a UAV self-stabilization algorithm. Node layout is generated using Gephi software with energy-model layout algorithm Force Atlas 2. Nodes are color-coded based on the communities identified by (a) ORC and (b) METIS algorithms, respectively.

Definition 4.3. A quality function determines how efficient the LLVM IR instructions are grouped in terms of energy consumption, parallelism, load balancing, hardware utilization, and inter-cluster data movements.

The discovery of the processing community structure problem can now be formulated as follows: given a DDG, find non-overlapping subgraph cut which maximize the quality function:

$$
Q=\sum_{c=1}^{n_{c}}\left(\frac{\left(W_{c}-S_{c}\right)}{W}-\frac{\left(W_{c}-\bar{W}\right)^{2}}{W}\right),
$$

and satisfy:

$$
N \geq n_{c},
$$

where $n_{c}$ denotes the number of clusters; $W_{c}$ stands for the sum of edge weights within cluster $c$; $W$ is the sum of all edge weights; $\bar{W}$ is the average sum of edge weights among all the clusters; $S_{c}$ represents the sum of edges weights connected to cluster $c$; and $N$ is the core count. The first term in Equation (5) confines the data flow within the cluster as much as possible. It indicates the difference between the sum of the weights in a cluster and the sum of the weights of the edge connected to the cluster. The greater this term is, the fewer inter-cluster data movements, and the more energy is saved. The second term in Equation (5) measures the standard deviation squared between sum of weights in cluster $c$ and average sum of weights in all clusters. Minimizing this term ensures load balancing and fully takes advantage of parallel execution.

While optimal communities discovery is an Non-deterministic Polynomial-time (NP)-hard problem, we use the ORC-based community detection algorithm [34] to decide which node should be grouped in which cluster. The complexity of this algorithm is $O\left(E D^{2}\right)$, where $E$ is the number of edges in DDG, and $D$ is the average degree. The algorithm is consisted of the following steps: (1)it calculates the ORC values for all edges in the network representation of the high-level codes; (2) it removes the edge with the most negative ORC value; (3) it re-calculates the edge ORC value only for those affected nodes/edges due to prior edge removals; (4) it checks if all edge curvature values are non-negative, otherwise it repeats steps 2 and 3 until there is no negatively curved edges; and (5) it performs a preferential attachment of the isolated graph components if the sizes of the communities are imbalanced.

In this example, the graph contains 724 nodes and 1,025 edges with an average degree of 1.4. Figure 8 shows the communities identified by the ORC- and METIS-based community detection algorithms, respectively. The ORC-based algorithm initially identifies 40 communities of which 27 of them have sizes smaller than $3 \%$ of the graph size. Setting the total number of communities to be 16 and the minimum community size to be $3 \%$ of the graph size, we can apply a preferential 


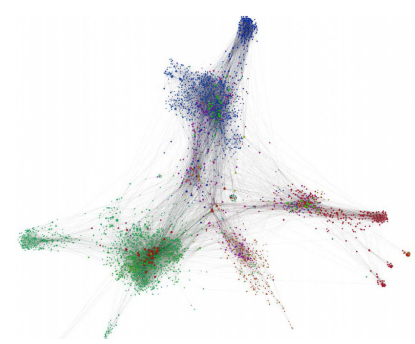

(a) Graph representation of the flight controller.

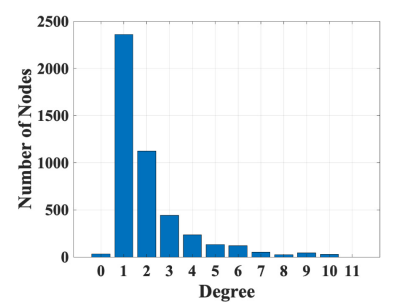

(d) Degree distribution of the flight controller.

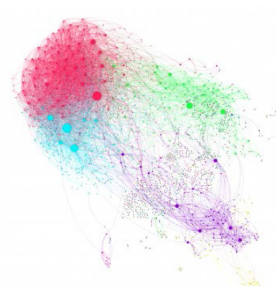

(b) Graph representation of the path planning algorithm.

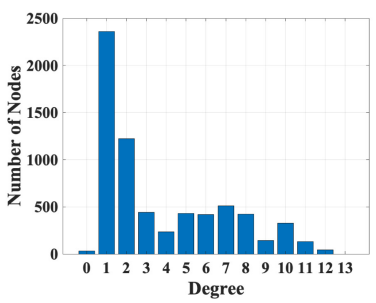

(e) Degree distribution of the path planning.

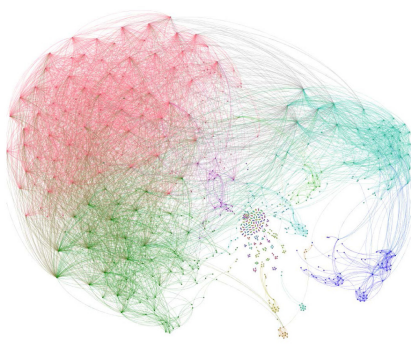

(c) Graph representation of the visual navigation.

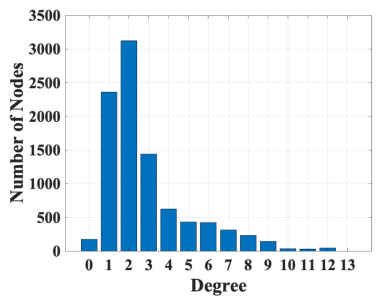

(f) Degree distribution of the visual navigation.

Fig. 9. Graph models and degree distributions of UAV applications.

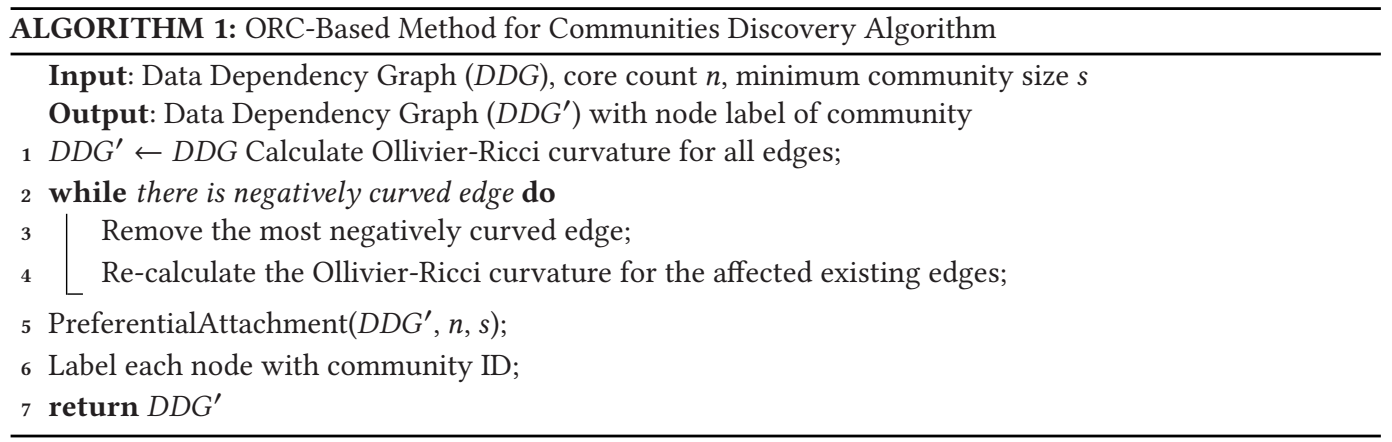

attachment based on the inter-community ORC to merge those small communities into larger ones. The 16 communities identified by Algorithm 1 are color-coded as shown in Figure 8(a). On the other hand, the METIS-based CI is able to identify 13 communities as shown in Figure 8(b). For this particular graph, when applied Equation (5), the ORC approach achieves a quality value of 1,782, which beats METIS's 982 . The fewer the inter-cluster edge weights produced by ORC will entail less communication overhead when running on the NoC, which reduces the dynamic energy. In addition, ORC obtains a smaller variation of clusters' sizes, which means better load balance among cores and saves energy as well.

Figures 9(a)-(c) show the DDG connectivity structure for several UAV applications (e.g., the flight controller, the path planning, and the visual navigation module of a UAV). Figures 9(d)-(f) summarize their degree distributions in order to investigate their topological complexity and understand whether advanced graph and differential geometry techniques like the ones developed 
in this work may provide good results. As we can observe from all three degree distribution plots, the different intelligence modules of the UAV show distinct features and we need to develop a robust and rigorous design methodology capable to mine the topology of the weighted graphs corresponding to these high-level codes, identify the best strategies for parallelization and track these interdependencies among the identified tasks/threads in an automatic manner without programmer intervention; also we exploit this for hardware investigation and could be exploited in the future for additional tailoring of the architecture to include ASICs. In Figure 9, the DDGs can be classified into two categories: high and medium discernibility. The flight controller is a highly discernible DDG, where clusters are clearly separated. Medium discernible DDGs may be seen as some regular patterns by humans. The path planning and visual navigation are of medium discernibility, where the inter-cluster edges are more than the high discernible DDGs. The similarity in high and medium discernible DDGs is that there are some visible patterns, making them suitable for parallel execution. The reason is that array declaration in $\mathrm{C} / \mathrm{C}++$ programs corresponds to an IR instruction called "getelementptr". ${ }^{3}$ Therefore, all array-related operations depend on this instruction. This node is becoming central and the betweenness is very high due to array operations. One cluster should be centered on at least one of those nodes. As we can see in Figure 9(a), we can infer that there are at least five distributed clusters and operations on one cluster barely depend on those on another clusters.

\subsection{Compact Intelligence Mapping into Constrained Hardware}

The tile to which each cluster is mapped significantly affects the power consumption of the application since it determines the dynamic and static communication cost. Consequently, an approach, which is similar to the one in [19], is proposed, but it takes cluster ordering into consideration as well so that it reduces static energy consumption caused by congestion and contention of hardware resources.

Definition 4.4. A task graph (TG) is a weighted directed acyclic graph $T G=G\left(c_{i}, a_{i j}, v\left(a_{i j}\right)\right.$, $\left.b\left(a_{i j}\right)|i, j \in N|\right)$ where each vertex $c_{i}$ represents a cluster of LLVM IR instructions that are grouped together by our community detection algorithm, and each edge $a_{i j}$ represents communication from node $c_{i}$ to node $c_{j}$.

$-v\left(a_{i j}\right):$ data size from $c_{i}$ to $c_{j}$.

$-b\left(a_{i j}\right)$ : bandwidth requirement from $c_{i}$ to $c_{j}$.

Definition 4.5. An architecture graph (AG) is a directed graph $\left.A G=G\left(t_{i}, p_{i j}, e\left(p_{i j}\right)\right)|i, j \in N|\right)$ where each vertex $t_{i}$ represents a tile, and each edge $p_{i j}$ represents a routing path from $t_{i}$ to $t_{j}$.

$-e\left(p_{i j}\right)$ : energy consumption from $t_{i}$ to $t_{j}$.

$-L\left(p_{i j}\right)$ : set of links that makes up $p_{i j}$

The communication-weighted mapping (CWM) proposed in [19] fails to consider the order of the clusters, leading to significant potential congestion, and static energy consumption in NoCs. We illustrate the process of our communication dependency mapping (CDM) algorithm by mapping the TG in Figure 10(a) to the AG in Figure 10(b). The numbers attached to the links in Figure 10(b) are the corresponding packet sizes indicated in Figure 10(a). For example, the packet $A \rightarrow D$ is of size 8 , so the link $\mathrm{AD}$ has a value of 8 . At the same time, because a packet of 6 flits is

\footnotetext{
${ }^{3}$ The "getelementptr" instruction is used to get the address of a sub-element of an aggregate data structure. It performs address calculation only and does not access memory. The instruction can also be used to calculate a vector of such addresses [20].
} 


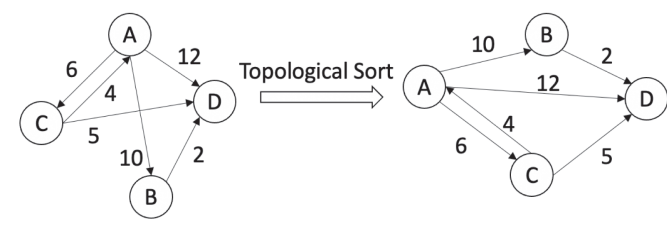

(a) Application of a topological sort to task graph.

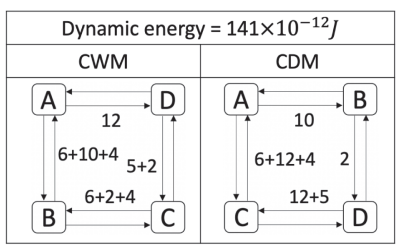

(b) Dynamic energy

Fig. 10. Mapping comparison: dynamic energy.

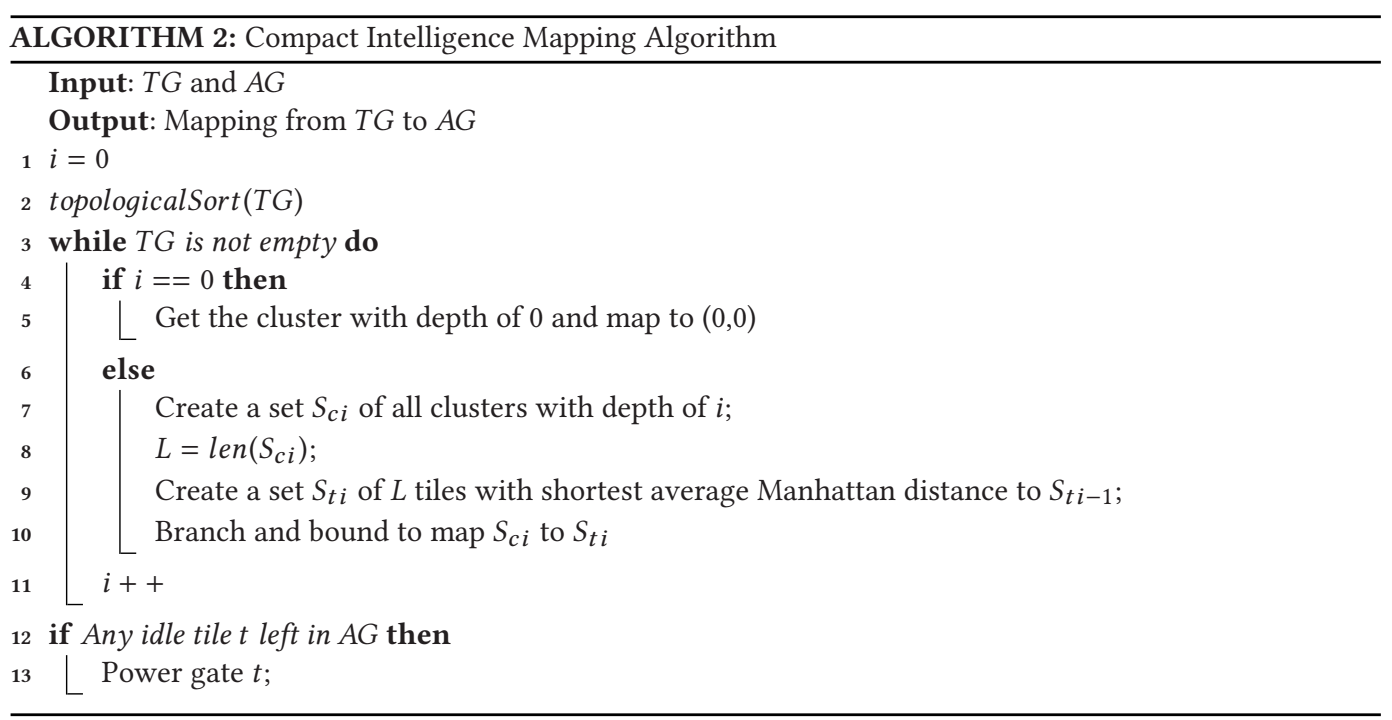

sent from $\mathrm{A}$ to $\mathrm{C}$, the link $\mathrm{AB}$ (multiplexed by the packet $A \rightarrow B$ ) and the link $\mathrm{BC}$ (multiplexed by the packet $B \rightarrow C$ ) have 6 added to their values.

Algorithm 2 presents the pseudocode of our CDM mapping algorithm. First, the topological sort is applied to the TG before mapping. The depth of cluster $c_{i}$ is defined as the maximum number of edges from the root to $c_{i}$. For example, the depth of cluster $D$ is 2 instead of 1 . In case of a loop, the depth is calculated without revisiting any clusters. In Figure 10(a), cluster $D$ cannot execute before cluster $B$ and $C$ because it needs data from both of them, while cluster $B$ and $C$ can execute in parallel because they are at the same depth. Second, a set $S_{c 1}$ of all clusters at depth 1 is created, which in this case is $\{B, C\}$. Since there are two clusters at depth 1 , a set $S_{t 1}$ of two tiles with the closest Manhattan distance to $(0,0)$ is created. In this case, $S_{t 1}=\{(1,0),(0,1)\}$. The branchand-bound $(\mathbf{B} \& \mathbf{B})$ algorithm of traditional CWM is applied to map $S_{c 1}$ to $S_{t 1}$ to find the most energy-aware mapping. By dividing the mapping problem into sub-problems at distinctive depths, and conquering one at a time, the search space of the $B \& B$ tree is significantly reduced. The same procedure is repeated until the $T G$ is empty. Finally, any idle tile will be power gated.

To show the potential congestion incurred by CWM, we apply both CWM and CDM to the TG in Figure 10(a) and obtain the following two different mappings in Figure 10(b). For illustration purposes, we assume $E_{S_{b i t}}=E_{L_{b i t}}=1 \times 10^{-12} \mathrm{~J} / \mathrm{bit}$. For instance, using Equation (2), the 6-flit packet from A to $C$ consume $E_{D y_{A C}}=6 \times\left(3 \times E_{S_{b i t}}+(3-1) \times E_{L_{b i t}}\right)=30 \times 10^{-12} \mathrm{~J}$ of dynamic power. Applying the same procedure, it can be calculated that both mappings' dynamic energy costs are $141 \times 10^{-12} \mathrm{~J}$. Since CWM focuses on minimizing the total dynamic energy, it is possible 


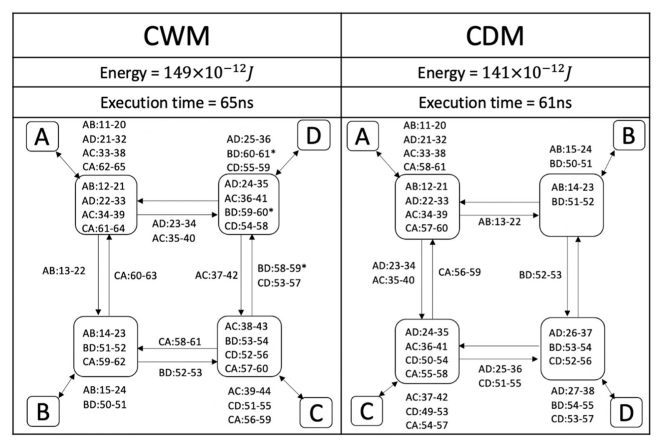

(a)

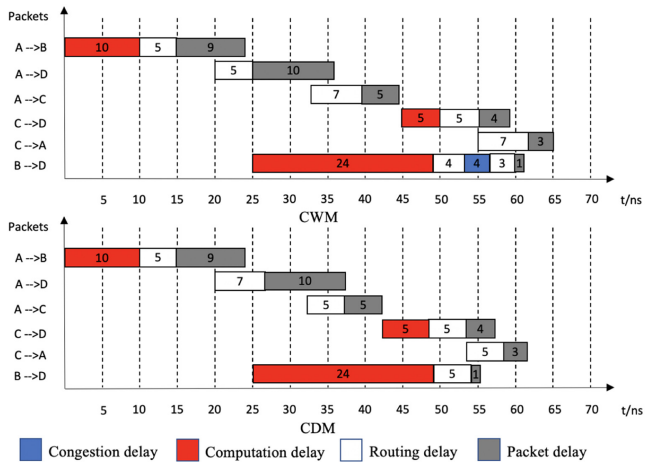

(b)

Fig. 11. Mapping comparison: static energy.

for it to place cluster $C$ two links away from cluster $A$. On the other hand, because CDM maps $\{B, C\}$ onto $\{(0,1),(1,0)\}$ in the same iteration, $C$ will be placed closer to $A$.

In terms of static energy, we assume $P_{S t}=1 \times 10^{-12} \mathrm{~J}$, and the execution time of cluster $\mathrm{A}$ is $10 n s$ while clusters $\mathrm{B}$ and $\mathrm{C}$ have execution time of $5 n s$ and $24 n s$, respectively. Also assume one packet flit is 1 bit and the time for a flit to pass through a switch $\left(t_{s}\right)$ is $1 n s$ and a link $\left(t_{l}\right)$ is $1 n s$.

Figure 11(a) evaluates the mapping results generated by CWM and CDM. Each edge and each box are annotated with the data communication in a given time interval. For example, the link between core $\mathrm{A}$ and its router is annotated with $A B: 11-20$, which means there is a packet from A to B occupying the link from 11ns to 20ns. As shown in Figure 11(a), the CWM's result causes conflict during $B D$ and $C D$ transmission. When the packet $C D$ uses the link between $C$ and $\mathrm{D}$, packet $B D$ is stored in the buffer of the router of core $\mathrm{C}$, waiting for the link to be cleared and consuming static energy. The delayed packet is marked with “*” in Figure 11(a). On the other hand, CDM's result does not incur any congestion because cluster B and C are of the same depth and are mapped closed to A.

Figure 11(b) shows the timing diagram of all computations and all packet deliveries of both mappings. Different kinds of delay are color-coded. For instance, in CWM, the first flit of the packet from cluster $A$ to $B$ takes $2 \times t_{s}+t_{l}=5 n s$ to arrive (routing delay), while the rest of the packet needs another $9 n$ s (packet delay). The congestion delay of packet BD is indicated as blue. Since there is resource contention in CDM, the execution time is reduced by $6.1 \%$ compared to CWM. In addition, the static energy overhead caused by the congestion delay in CWM is $E_{S t N o C}=$ $P_{S t} \times 2 \times 4=8 \times 10^{-12} \mathrm{~J}$, which means CWM consumes $5.67 \%$ more energy in interconnect.

\section{EXPERIMENTAL RESULTS}

In this section, we first evaluate and compare our ORC-based scheduling with Greedy Scheduling, Local Search, and Hierarchical Hungarian Scheduling in terms of the runtime speedup. Meanwhile, we examine our CDM mapping against CWM in terms of energy consumption. Finally, we run multiple UAV applications with our optimization framework on multicore system to demonstrate how the addition of cores affects the performance of the designed system.

\subsection{Experiment Setup}

We use gem5 [5] together with McPAT [21] for architectural and power simulation. Our hardware model is an Advanced RISC Machines (ARM) processor connected in a 2D mesh topology NoC from 4 to 128 cores [2] with $32 \mathrm{~KB} \mathrm{L1}$ cache as shown in Table 2. Detailed parameters are listed in Table 2. 
Table 2. Parameters of Simulation Processors

\begin{tabular}{|c|c|}
\hline Cores & Up to 128 cores in-order ARM cores at $500 \mathrm{MHz}$ \\
\hline L1 Private Cache & 32 KB, 4-way, 32-byte block \\
\hline Topology & 2D Mesh with XY routing \\
\hline
\end{tabular}

Table 3. Benchmarks and Their DDG Information

\begin{tabular}{|c|c|c|c|c|}
\hline Benchmark & No. of Nodes & No. of Edges & Avg. Degree & Avg. Weight \\
\hline Quadrotor Controller ${ }^{4}$ & 402,399 & 634,104 & 2.398 & 12.427 \\
\hline Nearest $^{5}$ & 729,422 & 929,243 & 2.734 & 13.432 \\
\hline 2-Opt Swap ${ }^{6}$ & 334,399 & 493,135 & 2.143 & 9.514 \\
\hline Random PST $^{7}$ & $1,348,311$ & $1,531,345$ & 2.434 & 7.358 \\
\hline ConvNet [33] & $2,243,142$ & $3,143,113$ & 2.922 & 19.343 \\
\hline
\end{tabular}

We conduct experiments on five benchmarks. Table 3 illustrates the detailed information of the DAG of each benchmark. "No. of nodes" represents the number of LLVM instructions in one iteration of each benchmark; "No. of Edges" stands for the number of dependency between all the LLVM instructions in one iteration. "Avg. Degree" and "Avg. Weight" indicate the average degree of each node and the average weight of each edge, respectively.

\subsection{Results and Discussion}

First, we compare our community discovery algorithm with a range of existing scheduling algorithms, including the maximum permissible task assignment (MPTA) [8], the Iterative Offline Energy-Aware Task and Communication Scheduling (IOETCS) [36], and Hierarchical Hungarian [14] by running a flight controller application. Figure 12(a) examines our ORC-based community discovery algorithm's (Algorithm 1) computational complexity as the number of core grows. The processing community discovery is done offline (only once), so the run time will not affect the controller speed during UAV navigation. Figure 12(a) shows the run time overhead of the referenced scheduling against our algorithm over a range of 4-128 core organizations. For a small number of cores, the run time overhead is acceptable, but increases rapidly for the MPTA and Hierarchical Hungarian. Hierarchical Hungarian has $O\left(n^{2}\right)$ complexity. The timing complexity of MPTA is $O\left(n^{3} P_{\max }\left(\begin{array}{c}n+C_{\max } \\ C_{\max }\end{array}\right)\right)$, where $n$ is the number of tasks, $P_{\max }$ is the scheduling period, and $C_{\max }$ is the largest WCET. The complexity explodes as soon as $n$ becomes large. Meanwhile, the IOETCS has a polynomial time complexity.

When using the same mapping scheme, Figure 12(b) evaluates the performance loss of the various scheduling algorithms over our ORC-based communities discovery algorithm. The IOETCS exhibits the largest performance loss of roughly $9 \%$ for large core count. This is because it fails to consider the complexity of scheduling while only focusing on ranking applications by Instruction Per Clock (IPC). A particular application may suffer significantly if one core has much lower IPC than others. The Hierarchical Hungarian delivers a decent performance as the core count increases while the MPTA offers the best combination of run time and performance. However, they both struggle to detect small clusters compared to the graph as a whole because they have a resolution limit. The ORC-based algorithm is able to discover finer hierarchical structures of

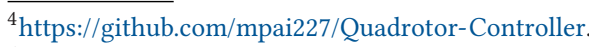

${ }^{5}$ https://github.com/rayjyh/traveling-salesman.

${ }^{6}$ https://github.com/rayjyh/traveling-salesman.

${ }^{7}$ https://github.com/rayjyh/traveling-salesman.
} 


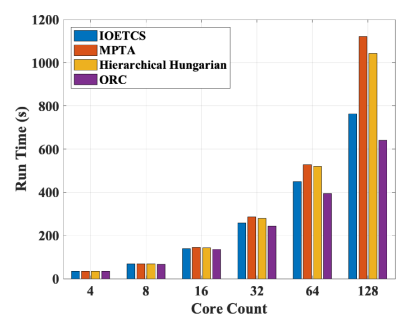

(a) Scheduling overheads.

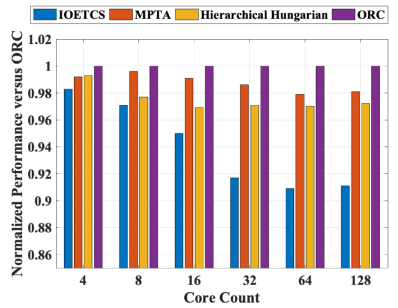

(b) Scheduling performance.

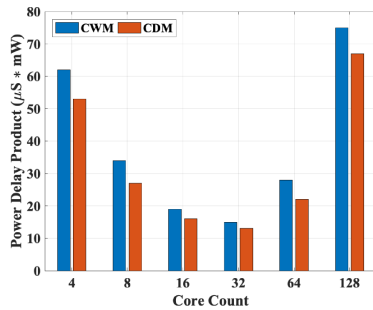

(c) Energy comparison.

Fig. 12. UAV flight controller scheduling results.

the network, it identifies better instruction parallelism within the densely-connected community structure and minimizes the communication between clusters.

Next, we compare the energy consumption of our CDM and CWM mapping in Figure 12(c). Both mappings use the same communities generated by our ORC clustering algorithm. The power values are collected by feeding the outputs from gem5 to McPAT. Having fully taken advantage of parallel execution, load-balancing, and optimal inter-community communication, our design has achieved an average of $17 \%$ less Power Delay Product over CWM as the maximum Power Delay Product (PDP) saving of $22 \%$ is achieved for 64-core configuration. As the core count grows, the PDP decreases significantly from 4-core to the lowest at 32 -core for $76 \%$. Starting from 64 cores, the increase of power consumption becomes the dominant factor and the number of flits needed to be routed between cores soars as PDP increases significantly. The lowest PDP is achieved by a 32 -core configuration at $13.7 \mu S * m W$. The reason why PDP decreases first is that from 4-core to 32-core the reduction of delay thanks to the parallel execution and fewer inter-core communication overshadows the increase of the power consumption caused by the addition of cores. Beyond 32-core, the increase of power consumption becomes the dominant factor of PDP and the number of flits to be routed soars.

In addition, we test our ORC-based scheduling and energy-aware mapping on 32-core configuration with the various map sizes and obstacle percentages. Figures 13(a)-(b) scale the map size between $10 \times 10$ and $50 \times 50$ while the percentage of obstacles is fixed at $20 \%$. When using the same mapping scheme, our ORC-based scheduling has shown an average of $5 \%$ flight time reduction compared to the second-best algorithm. On the other hand, CWM consumes up to $10.9 \%$ less energy than CDM when they share the same scheduling algorithm. In Figures 13(c)-(d), the map size is fixed at $50 \times 50$ and the obstacles increments from $0 \%$ to $30 \%$ with a $10 \%$ interval. The baseline that uses the combination of MPTA scheduling and CWM mapping benefit less from the parallel optimization because of the low efficiency of data migration between cores. As expected, we observe an average of $23 \%$ flight time reduction and up to $34 \%$ energy saving.

Finally, we apply our optimization framework to two other major UAV applications, such as path planning with timing constraints and visual navigation. After studying the state of the art of drone flight controllers and autopilot [11], we configure the baseline as a single-core ARM CPU. Although it is not fair to compare multicore to single-core to justify performance speedup, it is a good representative for the existing industrial solutions, as most of them are built upon an STM32 Microcontroller Unit (MCU).

We validate our platform by running three TSP algorithms in a simulated area of $100 \times 100$ discretization size with 50 randomly placed nodes. Each node is given a visit deadline randomly generated between 30 and 90 seconds. We evaluate the efficiency of the algorithm in terms of on-time visited node percentage and energy consumption of the flight. In Figure 14, our optimization methodology on multicore platforms outperforms the baseline when running all 


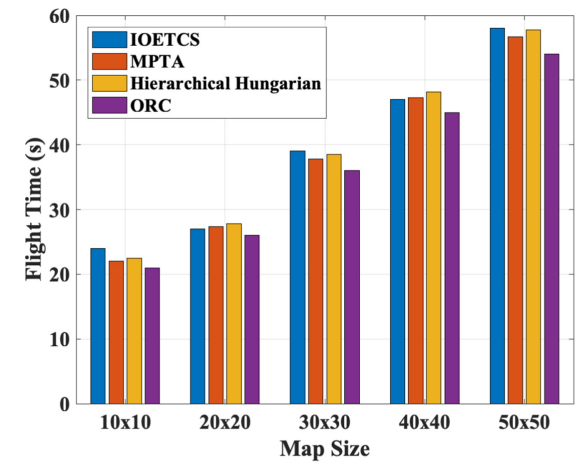

(a) Flight time comparison of different scheduling algorithms.

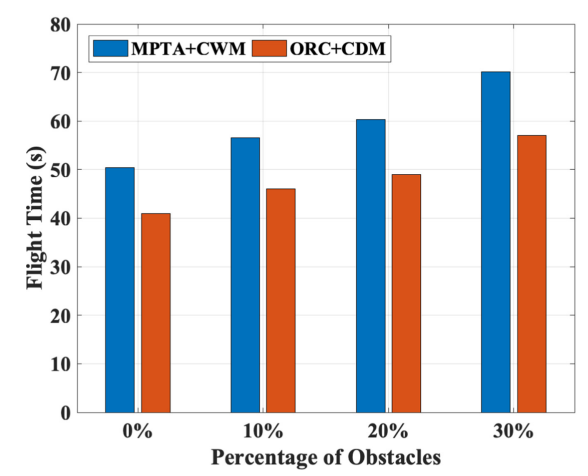

(c) Flight time comparison under different percentages of obstacles.

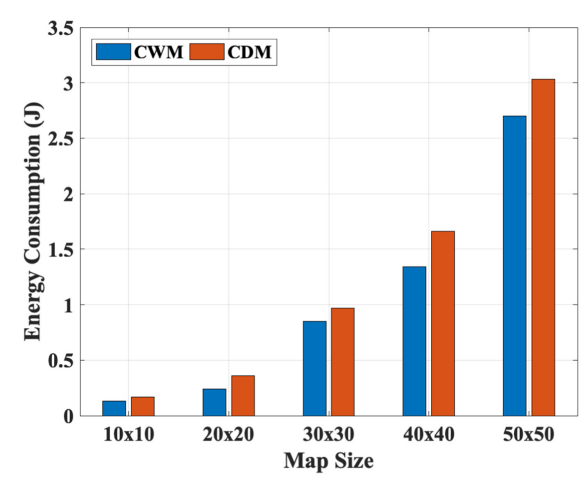

(b) Energy consumption comparison of different mapping algorithms.

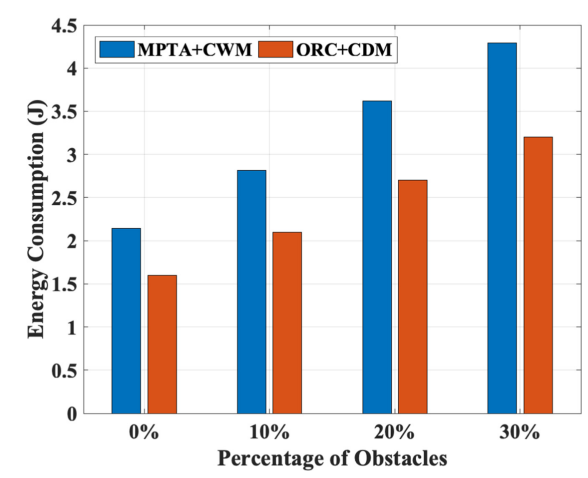

(d) Energy consumption comparison under different percentages of obstacles.

Fig. 13. Energy consumption and flight time over different map sizes and obstacle percentages.

three TSP algorithms. The largest on-time visit rate improvement of $14 \%$ is achieved when running Random TSP. On the other hand, the 16-core solution provides the best energy efficiency overall TSP algorithms. The serial execution of the baseline model incurs high travel time, making it less likely to visit more nodes in a tight time frame.

$\mathrm{CNN}$ is the building block of the DroNet which is widely-used in visual navigation for UAVs. We run a widely-used ConvNet benchmark with $32 \times 32$ input images on both our optimized platform and baseline machine to compare the performance and energy consumption difference. The CNN has 2,200 parameters and a footprint of 4,400 bytes on L2 memory. We use 16-bit fixedpoint numbers for inputs, kernels, and outputs. And we use $3 \times 3$ kernels. Figure 15(a) shows the performance of ConvNet when scheduled on baseline, 4, 8, 16, and 32 cores. Since ConvNet is a highly parallel algorithm, execution time measured in cycles decreases almost linearly with the number of cores. The fastest performance is achieved at 32-core, which is $81 \%$ run time reduction compared to the baseline configuration. Figure 15(b) shows the energy efficiency of the ConvNet on a frame when scaling between baseline, 4, 8, 16, and 32 cores. The energy/performance tradeoff is illustrated: a peak performance of 55 FPS can be delivered by 32 -core at a cost of $16.4 \mathrm{~mJ}$ per frame. Whereas the baseline can process no more than 21 frames per second at a cost of $33.3 \mathrm{~mJ}$ per frame. 


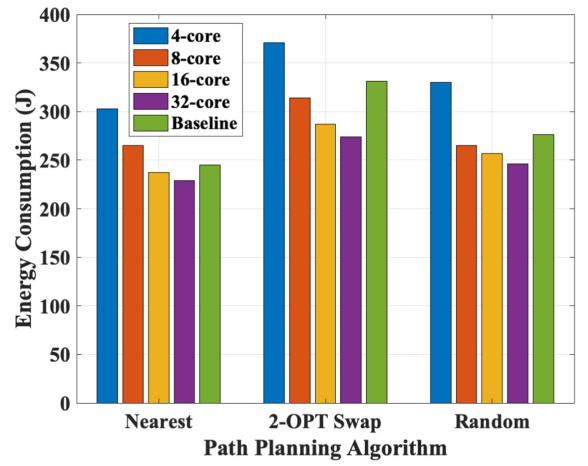

(a) Energy consumption comparison of different core counts.

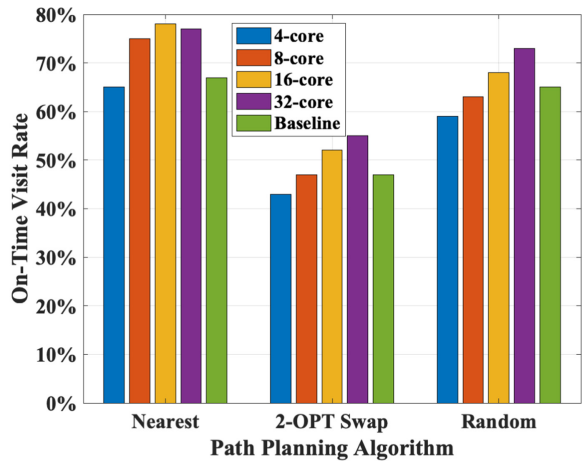

(b) On-time visit rate comparison of different core counts.

Fig. 14. Energy consumption and on-time visit rate over different TSP algorithms.

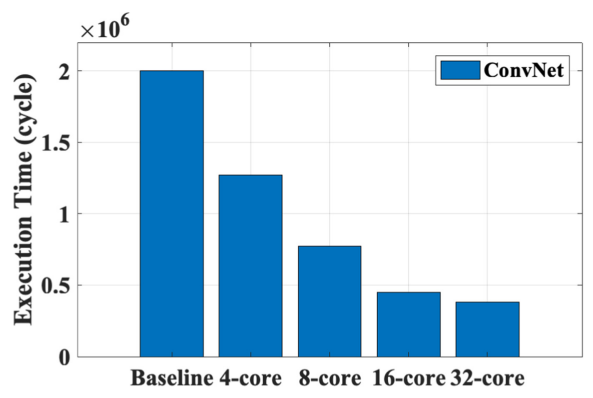

(a) Execution time of different core counts.

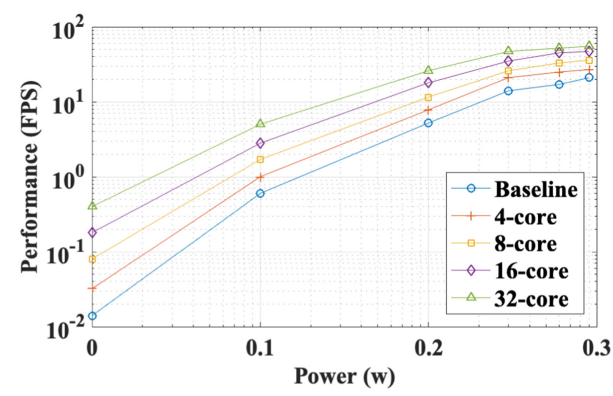

(b) Energy efficiency of different core counts.

Fig. 15. ConvNet execution time and energy efficiency on different core counts.

\section{CONCLUSION}

In this article, we develop a two-stage optimization framework for drone PNI applications. First, we compile a PNI application into LLVM IR, from which a DDG is constructed. We then analyze the DDG structure and discover its best parallelization degree by applying our load-balancing ORC-based scheduling algorithm so that data movement is confined within clusters. Finally, a congestion-aware mapping scheme based on the topological sort is proposed to map clusters onto the NoCs for parallel execution. The experimental results show that the proposed framework achieves $23 \%$ flight time reduction and up to $34 \%$ energy saving for flight controller. In addition, the optimization on 16-core platform improves the on-time visit rate of the path planning algorithm by $14 \%$ while reducing $81 \%$ of run time for ConvNet visual navigation.

\section{REFERENCES}

[1] S. Kumar, A. Jantsch, J.-P. Soininen, M. Forsell, M. Millberg, J. Oberg, K. Tiensyrja, and A. Hemani. 2002. A network on chip architecture and design methodology. In Proceedings of the IEEE Computer Society Annual Symposium on VLSI. IEEE Computer Society, 117.

[2] Niket Agarwal, Tushar Krishna, Li-Shiuan Peh, and Niraj K. Jha. 2009. GARNET: A detailed on-chip network model inside a full-system simulator. In 2009 IEEE International Symposium on Performance Analysis of Systems and Software. IEEE, 33-42.

[3] Stephen Armah, Sun Yi, Wonchang Choi, and Dongchui Shin. 2016. Feedback control of quad-rotors with a matlabbased simulator. American fournal of Applied Sciences 13, 6 (2016), 779-793. 
[4] Serhiy Avramenko, Stefano Esposito, Massimo Violante, Marco Sozzi, Massimo Traversone, Marco Binello, and Marco Terrone. 2015. An hybrid architecture for consolidating mixed criticality applications on multicore systems. In Proceedings of the 2015 IEEE 21st International On-Line Testing Symposium. IEEE, 26-29.

[5] Nathan Binkert, Bradford Beckmann, Gabriel Black, Steven K. Reinhardt, Ali Saidi, Arkaprava Basu, Joel Hestness, Derek R. Hower, Tushar Krishna, Somayeh Sardashti, Rathijit Sen, Korey Sewell, Muhammad Shoaib, Nilay Vaish, Mark D. Hill, and David A. Wood. 2011. The Gem5 simulator. ACM SIGARCH Computer Architecture News 39, 2 (2011), 1-7.

[6] Paul Bogdan. 2015. Mathematical modeling and control of multifractal workloads for data-center-on-a-chip optimization. In Proceedings of the 9th International Symposium on Networks-on-Chip. ACM, 21.

[7] Paul Bogdan and Yuankun Xue. 2015. Mathematical models and control algorithms for dynamic optimization of multicore platforms: A complex dynamics approach. In Proceedings of the IEEE/ACM International Conference on ComputerAided Design. IEEE, 170-175.

[8] Jinchao Chen, Chenglie Du, Fei Xie, and Bin Lin. 2018. Scheduling non-preemptive tasks with strict periods in multicore real-time systems. fournal of Systems Architecture 90, 1 (2018), 72-84.

[9] Peter Corke. 2017. Flying Robots Book: Robotics, Vision and Control. Springer.

[10] William J. Dally and Brian Towles. 2001. Route packets, not wires: On-chip inteconnection networks. In Proceedings of the 38th Annual Design Automation Conference. ACM, 684-689.

[11] Emad Ebeid, Martin Skriver, Kristian Husum Terkildsen, Kjeld Jensen, and Ulrik Pagh Schultz. 2018. A survey of open-source UAV flight controllers and flight simulators. Microprocessors and Microsystems 61, 1 (2018), 11-20.

[12] Stefano Esposito and Massimo Violante. 2017. On the consolidation of mixed criticalities applications on multicore architectures. Fournal of Electronic Testing 33, 1 (2017), 65-76.

[13] Santo Fortunato. 2010. Community detection in graphs. Physics Reports 486, 3-5 (2010), 75-174.

[14] Jacob Goldberger and Tamir Tassa. 2008. A hierarchical clustering algorithm based on the Hungarian method. Pattern Recognition Letters 29, 11 (2008), 1632-1638.

[15] G. Guindani and F. G. Moraes. 2013. Achieving QoS in NoC-based MPSoCs through dynamic frequency scaling. In Proceedings of the 2013 International Symposium on System on Chip. 1-6. DOI : https://doi.org/10.1109/ISSoC.2013.6675275

[16] K. He, X. Zhang, S. Ren, and J. Sun. 2016. Deep residual learning for image recognition. In Proceedings of the 2016 IEEE Conference on Computer Vision and Pattern Recognition. 770-778.

[17] Karla L. Hoffman, Manfred Padberg, and Giovanni Rinaldi. 2013. Traveling salesman problem. In Encyclopedia of Operations Research and Management Science. S. I. Gass and M. C. Fu (Eds.), Springer, Boston, MA, 1573-1578. DOI : https://doi.org/10.1007/978-1-4419-1153-7_1068

[18] Gabriel Hoffmann, Haomiao Huang, Steven Waslander, and Claire Tomlin. 2007. Quadrotor helicopter flight dynamics and control: Theory and experiment. In Proceedings of the AIAA Guidance, Navigation and Control Conference and Exhibit. 6461.

[19] Jingcao Hu and Radu Marculescu. 2003. Exploiting the routing flexibility for energy/performance aware mapping of reular NoC architectures. In Proceedings of the 2003 Design, Automation and Test in Europe Conference and Exhibition. IEEE, 688-693.

[20] Chris Lattner and Vikram Adve. 2004. LLVM: A compilation framework for lifelong program analysis \& transformation. In Proceedings of the International Symposium on Code Generation and Optimization: Feedback-Directed and Runtime Optimization. IEEE Computer Society, 75.

[21] Sheng Li, Jung Ho Ahn, Richard D. Strong, Jay B. Brockman, Dean M. Tullsen, and Norman P. Jouppi. 2009. McPAT: An integrated power, area, and timing modeling framework for multicore and manycore architectures. In Proceedings of the 42nd Annual IEEE/ACM International Symposium on Microarchitecture. ACM, 469-480.

[22] Wuchen Li, Ernest Ryu, Stanley Osher, Wotao Yin, and Wilfrid Gangbo. 2018. A parallel method for Earth mover's distance. Fournal of Scientific Computing 75, 1 (2018), 182-197. DOI : https://doi.org/10.1007/s10915-017-0529-1

[23] Antonio Loquercio, Ana I. Maqueda, Carlos R. Del-Blanco, and Davide Scaramuzza. 2018. Dronet: Learning to fly by driving. IEEE Robotics and Automation Letters 3, 2 (2018), 1088-1095.

[24] R. Marculescu and P. Bogdan. 2009. The chip is the network: Toward a science of network-on-chip design. Foundations and Trendső in Electronic Design Automation 2, 4 (2009), 371-461. DOI : https://doi.org/10.1561/1000000011

[25] Radu Marculescu, Umit Y. Ogras, Li Shiuan Peh, Natalie Enright Jerger, and Yatin Hoskote. 2009. Outstanding research problems in NoC design: System, microarchitecture, and circuit perspectives. IEEE Transactions on Computer-Aided Design of Integrated Circuits and Systems 28, 1 (Jan. 2009), 3-21. DOI : https://doi.org/10.1109/TCAD.2008.2010691

[26] Mark E. J. Newman. 2003. The structure and function of complex networks. SIAM Review 45, 2 (2003), 167-256.

[27] Jan Nowotsch, Michael Paulitsch, Daniel Bühler, Henrik Theiling, Simon Wegener, and Michael Schmidt. 2014. Multicore interference-sensitive WCET analysis leveraging runtime resource capacity enforcement. In Proceedings of the 2014 26th Euromicro Conference on Real-Time Systems. IEEE, 109-118. 
[28] Jan Nowotsch, Michael Paulitsch, Arne Henrichsen, Werner Pongratz, and Andreas Schacht. 2014. Monitoring and WCET analysis in COTS multi-core-SoC-based mixed-criticality systems. In Proceedings of the 2014 Design, Automation \& Test in Europe Conference \& Exhibition. IEEE, 1-5.

[29] Daniele Palossi, Michele Furci, Roberto Naldi, Andrea Marongiu, Lorenzo Marconi, and Luca Benini. 2016. An energyefficient parallel algorithm for real-time near-optimal UAV path planning. In Proceedings of the ACM International Conference on Computing Frontiers. ACM, New York, NY, 392-397. DOI : https://doi.org/10.1145/2903150.2911712

[30] Sujit Rajappa, Markus Ryll, Heinrich H. Bülthoff, and Antonio Franchi. 2015. Modeling, control and design optimization for a fully-actuated hexarotor aerial vehicle with tilted propellers. In Proceedings of the 2015 IEEE International Conference on Robotics and Automation. IEEE, 4006-4013.

[31] Gerhard Reinelt. 1991. TSPLIB-A traveling salesman problem library. INFORMS fournal on Computing 3, 4 (Nov. 1991), 376-384. DOI : https://doi.org/10.1287/ijoc.3.4.376

[32] Mario Ruiz Estrada. 2020. The Uses of Drones in Case of Massive Epidemics Contagious Diseases Relief Humanitarian Aid: Wuhan-COVID-19 Crisis. Retrieved February 29, 2020 from https://doi.org/10.2139/ssrn.3546547

[33] Ali Sharif Razavian, Hossein Azizpour, Josephine Sullivan, and Stefan Carlsson. 2014. CNN features off-the-shelf: An astounding baseline for recognition. In Proceedings of the IEEE Conference on Computer Vision and Pattern Recognition Workshops. 806-813.

[34] Jayson Sia, Edmond Jonckheere, and Paul Bogdan. 2019. Ollivier-Ricci curvature-based method to community detection in complex networks. Scientific Reports 9, 1 (2019), 9800.

[35] Cheng Tan, Aditi Kulkarni, Vanchinathan Venkataramani, Manupa Karunaratne, Tulika Mitra, and Li-Shiuan Peh. 2018. LOCUS: Low-power customizable many-core architecture for wearables. ACM Transactions on Embedded Computing Systems 17, 1 (2018), 16.

[36] Umair Ullah Tariq, Hui Wu, and Suhaimi Abd Ishak. 2018. Energy-aware scheduling of conditional task graphs on noc-based mpsocs. In Proceedings of the 51st Hawaii International Conference on System Sciences.

[37] David Ullman, Vincent Homer, and Patrick Horgan. 2017. Comparing Electric Sky Taxi Visions. DOI : https://doi.org/ 10.13140/RG.2.2.14819.50729

[38] Chi Wang, Edmond Jonckheere, and Reza Banirazi. 2014. Wireless network capacity versus Ollivier-Ricci curvature under heat-diffusion (HD) protocol. In Proceedings of the American Control Conference, 3536-3541. D0I : https://doi. org/10.1109/ACC.2014.6858912

[39] Yao Xiao, Yuankun Xue, Shahin Nazarian, and Paul Bogdan. 2017. A load balancing inspired optimization framework for exascale multicore systems: A complex networks approach. In Proceedings of the 36th International Conference on Computer-Aided Design. IEEE, 217-224.

[40] Terry Tao Ye, Luca Benini, and Giovanni De Micheli. 2002. Analysis of power consumption on switch fabrics in network routers. In Proceedings of the 39th Annual Design Automation Conference. IEEE, 524-529.

Received January 2020; revised August 2020; accepted May 2021 